\title{
Simulación y análisis de inundaciones en cabeceras de cuenca, utilizando el software IBER en diferentes tramos
}

\author{
Simulation and analysis of floods in water basin heads, using the IBER \\ software in different sections
}

\author{
Francisco Alejandro Alcántara Boza ${ }^{1}$, Clever Torres Rojas ${ }^{2}$, Leonel Rolando Lucas Vidal ${ }^{3}$, \\ Johnny Daniel Bravo Loor ${ }^{4}$
}

Recibido: 27/08/2020 - Aprobado: 04/12/2020 - Publicado: 18/06/2021

\begin{abstract}
RESUMEN
El presente estudio se realizó en la confluencia de las microcuencas Baños, Minascocha y Quilla; que pertenece a la parte alta de la cuenca ChancayHuaral, con la finalidad de identificar las zonas de mayor vulnerabilidad frente a los fenómenos de pluviométricos de gran intensidad (inundaciones). Para la modelación de dicho tramo se utilizó los caudales que fueron obtenidos mediante el software SWAT. La simulación de la inundación se realizó mediante el software IBER V2.5, el cual es un software libre, que es utilizado en la simulación de inundaciones en aguas someras, es decir poco profundas. Los archivos utilizados fueron: imágenes satelitales, archivos DEM, caudales máximos. También se utilizó los Sistemas de Información Geográfica: ArcGis 10.5, QGIS 3.8.2, SASPLANET, GLOBALMAPER. La base matemática fueron las ecuaciones bidimensionales de St. Venant, el cual incorpora la turbulencia y rozamiento del flujo. Los resultados se muestran en tres puntos estratégicos a la salida de cabeceras de cuenca, con secciones transversales, velocidad, calado y froude. Como resultados se observa que en cabeceras de cuenca no se observa inundaciones, las velocidades del flujo de agua disminuyen en función al tiempo y distancia, ello debido a las características geomorfológicas encajonadas, el macizo rocoso, los sedimentos morrénicos a leptosoles y la vegetación herbácea - arbustiva.
\end{abstract}

Palabras claves: IBER; Geomorfología; Velocidad de flujo; Inundaciones; Cabecera de cuenca.

\begin{abstract}
The present study was carried out at the confluence of the Baños, Minascocha and Quilla micro-basins; which belongs to the upper part of the Chancay-Huaral basin, in order to identify the areas of greatest vulnerability to high intensity rainfall phenomena (floods). For the modeling of this section, the flows obtained by means of the SWAT software were used. The flood simulation was carried out using the IBER V2.5 software, which is free software, which is used to simulate floods in shallow waters, that is, shallow waters. The files used were satellite images, DEM files, maximum flows. The Geographic Information Systems were also used: ArcGis 10.5, QGIS 3.8.2, SASPLANET, GLOBALMAPER. The mathematical basis was the two-dimensional equations of St. Venant, which incorporates the turbulence and friction of the flow. The results are shown in three strategic points at the exit of the headwaters of the basin, with cross sections, speed, draft and froude. As results, it is observed that floods are not observed in the headwaters of the basin, the speeds of the water flow decrease as a function of time and distance, due to the confined geomorphological characteristics, the rocky massif, the moraine sediments to leptosols and the herbaceous vegetation - shrubby.
\end{abstract}

Keywords: IBER; geomorphology; maximum flows; floods; basin head.

\footnotetext{
1 Docente de la Universidad Nacional Mayor de San Marcos, Facultad de Ingeniería Geológica, Minera, Metalúrgica y Geográfica. Lima, Perú.

E-mail: falcantarab@unmsm.edu.pe - ORCID: https://orcid.org/0000-0001-9127-4450

2 Estudiante de la Universidad Nacional Mayor de San Marcos, Escuela Profesional de Ingeniería Geográfica. E-mail: clever.torres@unmsm.edu.pe

3 Docente de la Escuela Superior Politécnica Agropecuaria de Manabí-ESPAM. Ecuador.

E-mail: leonellucasvidal@espam.edu.ec ORCID: https://orcid.org/0000-0002-2286-9407

4 Docente de la Escuela Superior Politécnica Agropecuaria de Manabí-ESPAM. Ecuador. E-mail: jonisitobravol@hotmail.com
}

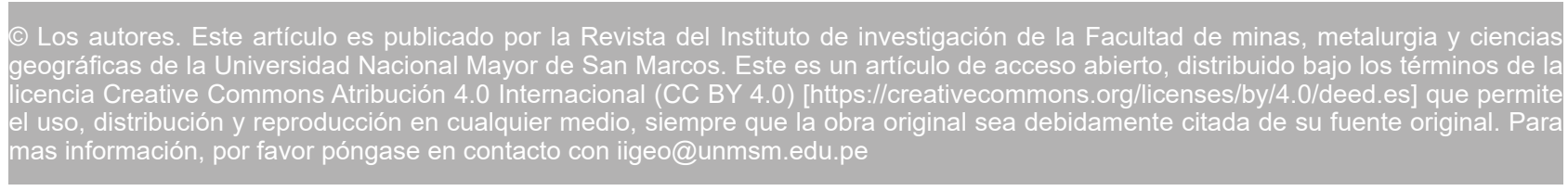




\section{INTRODUCCIÓN}

Los recursos hídricos, presentan un régimen de alteración cuantitativa a lo largo del tiempo. En Perú, tenemos dos épocas bien marcadas: altos caudales o crecidas (noviembre - abril) y mínimos caudales o estiaje (mayo a octubre). Por ende, el aumento del caudal en un río puede ser un riesgo para la población que se encuentra en los márgenes del cauce del río por ello es importante la modelización numérica (Bladé et al., 2014). Ante este problema y haciendo uso de la tecnología, es posible simular las inundaciones que depende del caudal de entrada, con la finalidad de predecir y evitar los desastres naturales en diferentes espacios de estudios, como lo presenta Martínez, (Martínez et al., 2017). Existen muchos softwares hidrológicos e hidráulicas; el software IBER, es un modelo hidráulico de simulación de inundaciones. El sistema de inundaciones es un fenómeno sin patrón de recurrencia con muy pocos estudios o simulaciones en cabeceras de cuenca por lo que la erosión del agua depende del enrutamiento (O'Connor et al., 2020)(O'Connor et al., 2020), y la geomorfología, importante en alta montaña; por lo que las inundaciones son más estudiadas en la parte media y baja de las cuencas (Sarango, D., Velásquez, T., Rozas, G., \& Gástelo, 2018).

IBER tiene la capacidad de simular los diferentes desastres que pudiesen ocurrir (inundaciones, erosión, sedimentación, entre otros), (Ochoa et al., 1999) (Martins et al., 2019). Por lo general, existe una tendencia de estudios más concurrentes en la parte baja de una cuenca o en un embalse (Sanz-Ramos et al., 2017), lo cual es lógico por el sistema de drenaje que presenta la misma. En nuestro caso se ha simulado la inundación a la salida de la microcuenca Baños que acumulan las aguas a su vez de otras microcuencas Baños, Minascocha y Quillas.

El área de estudio se encuentra en la parte alta de la cuenca Chancay-Huaral (Lima), de $2.48 \mathrm{~km}^{2}$ de análisis para la presente investigación; los suelos son leptosoles dístricos, suelos jóvenes de poco desarrollo genético según el mapa general de suelos del Perú; presentan un horizonte "A" poco desarrollado, los cuales se forman en pendientes convexas (Calzolari et al., 2021); además, con vegetación escasa, textura franco arenosa, con escasa retención de humedad y pendientes inclinadas, tendientes a la erosión (Poma \& Alcántara, 2011) (Ver figura 1).

Nuestra zona de origen de simulación se encuentra a $11^{\circ} 13^{\prime} 19.07^{\prime \prime}$ 'S y $76^{\circ} 36^{\prime} 11.78^{\prime \prime} \mathrm{O}$, a una altitud de 3580 msnm, la modelación hidráulica abarcó un trayecto de aproximadamente $2.3 \mathrm{~km}$, medida desde la confluencia de los ríos de las microcuencas Baños, Minascocha y Quilla. El área de influencia presenta una precipitación media de $600 \mathrm{~mm} / \mathrm{año}$, con un clima seco. La geología está constituido por andesitas, dacitas y riolitas la mayor parte de color pardo, marrón, así mismo lo constituyen los conglomerados (INGEMMET, 2020).

\section{MÉTODOS}

Los materiales base para la presente investigación, son las imágenes satelitales (descargado con el software de SASPLANET), cuya forma es rectangular y con una resolución de 0.29 pixeles, lo cual ayuda a la clasificación de los diferentes usos de suelo, y cuya delimitación se realizó con los Sistemas de Información Geográfica (SIG), específicamente con QGIS 3.8.2. Además, se utilizó un modelo digital de elevación (DEM) cuya descarga se realizó de la página del USGS - GLOVIS, de $12.5 \mathrm{~m}$ de resolución, ideal para pequeñas áreas de trabajo. En cuanto a los caudales (tabla 1) se obtuvo con al software hidrológico SWAT dato base para la simulación de inundación; los caudales para simulación correspondes desde 1990 al año 2005.

Las etapas de pre-procesamiento de los archivos del software IBER en la zona de estudio, se dividió en siete etapas: Primera parte: importación de datos y re-proyección; la imagen satelital (descargada desde SASPLANET), y el DEM (descargada de USGS GLOVIS), estos archivos se importó desde el software QGIS 3.8.2, en el cual se uniformizó las proyecciones mediante la herramienta proyectar al 3395 WGS84/ Word Mercator. Segunda parte: digitalización y exportación de archivos; sobre la imagen satelital se creó un archivo shapefile con la finalidad de delimitar los diferentes usos y tipos de suelos en la zona de estudio; en cuanto a la exportación de archivos, la imagen satelital se convirtió al formato .jpg (el cual es importable desde el interfaz de IBER) el mismo que ayudará a visualizar los resultados obtenidos. El siguiente formato, es la elevación de terreno en coordenadas (en formato .ASD), el cual ayudará a la modificación de la malla y la creación de secciones transversales y análisis en diferentes puntos del cauce del río. Tercera parte: Importación de archivos a IBER y creación de superficies; los archivos exportados y creados en el software QGIS 3.8.2 se importó desde el software IBER 2.5 (imágenes satelitales en formato.jpg y shapefile formato .shp), la creación de superficies se realizó en el archivo shapefile donde se designó de la rugosidad de acuerdo a los usos de suelo, creación de mallas y modificación de la malla esencial para tener resultados precisos (Gil \& Villanueva, 2010), (figura 2).

Cuarta parte: la asignación de diferentes rugosidades a la superficie de análisis, dado que el software tiene principios de hidráulica, por tanto, se debe evaluar el río. Para nuestro caso es un río Joven, con una tendencia en forma de V, cursos irregulares y contenido de material fracturado; con variaciones importantes en los niveles del lecho (Rocha, 2013) y con estabilidad Estática: Las corrientes arrastran sedimentos, pero, en el caso de ciertos tipos de ríos, no mueven ni arrastran partículas de las orillas, dado que las márgenes son rocosas o tienen alta cohesión (Rivera-Trejo et al., 2013). Luego, se utilizó la rugosidad de Manning para corrientes naturales, identificándose cauces limpios de bordes rectos con poca vegetación, así como cauces sinuosos de poco tirante con altas pendientes y rocoso. El criterio que se utilizó para la clasificación de la zona de estudio es el uso del suelo, lo cual se analizó mediante imágenes satelitales, determinando como suelos desnudos, areno-limoso, con arbustos y vegetación dispersa y rocas sinuosas. Respecto al caudal ingresado son las que se obtuvo mediante el software SWAT, (tabla 1).

Quinta parte: asignación de mallado y modificación del mallado; los ríos en general tienen una geometría irregular por lo que la construcción de una malla eficiente 

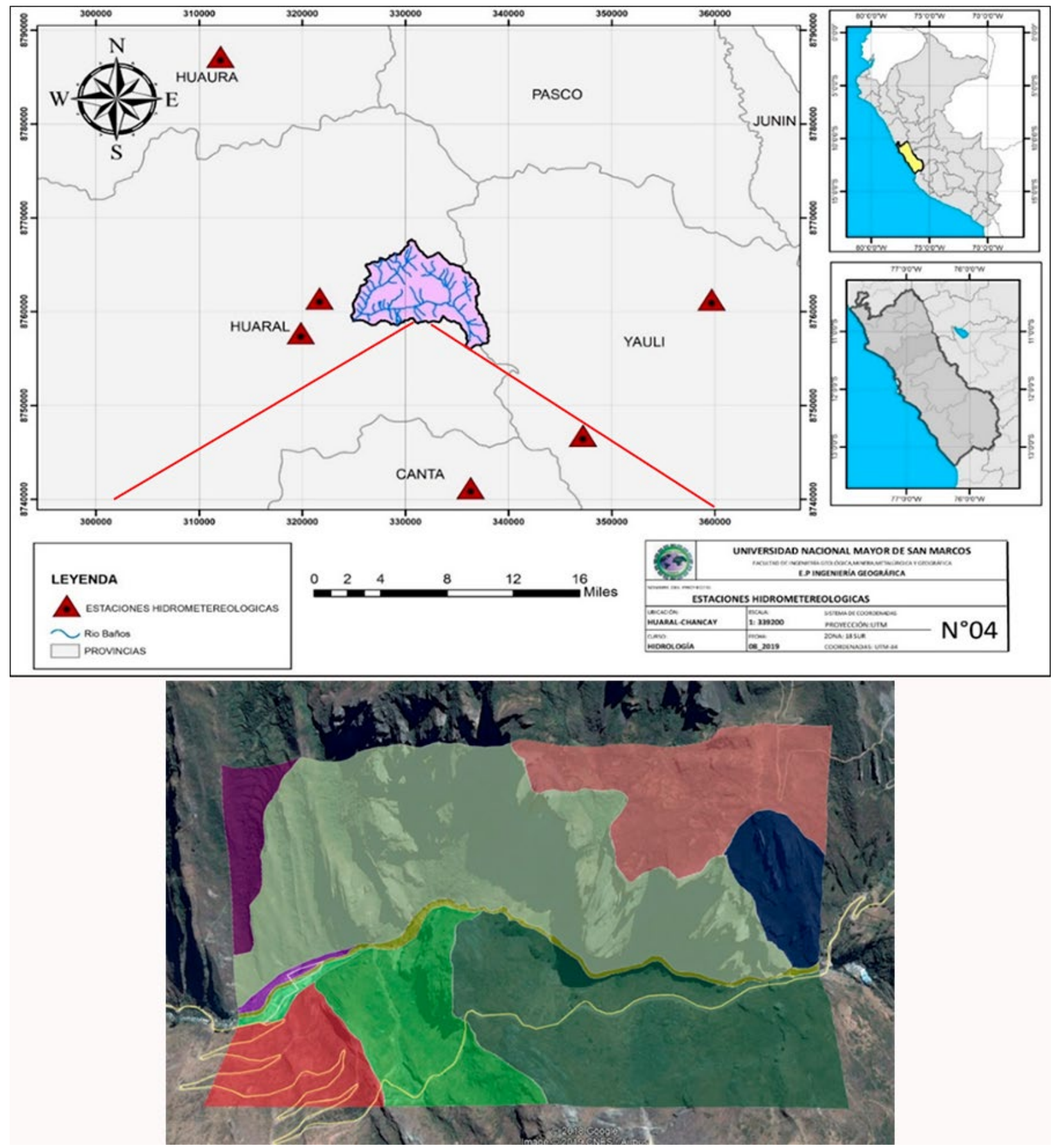

Figura 1. Ubicación del área de estudio a la salida de la microcuenca baños (Chancay-Huaral)

no es evidente. Es necesario que las mallas sean irregulares con el fin de minimizar los elementos con transiciones suaves, ello es esencial en la simulación (Areu-Rangel et al., 2019). IBER tiene la peculiaridad de creación de mallas estructuradas y no estructuras; para la zona de estudiada se eligió una malla no estructurada, al ser una topografía accidentada, (figura 3). La modificación de malla se puede realizar de diferentes formas como lo menciona, (Bladé et al., 2014).

Sexta parte: condiciones del problema y cálculo de los caudales en diferentes intervalos de tiempos; en esta parte se configuró el tiempo de inicio, tiempo máximo de simulación y el Intervalo de tiempo a evaluar, considerando un intervalo de tiempo de $7200 \mathrm{~s}$ y finalmente se procedió a calcular.

El modelo matemático que se utilizó para analizar los caudales se basa en las ecuaciones de St. Venant, bidimensionales incorporando los efectos de turbulencia y rozamiento superficial por el viento (Bladé et al., 2014) (figura 4). En dónde $h$ es el calado; $U x$ y $U y$ son las velocidades horizontales y promediadas en profundidad, $g$ es la aceleración de la gravedad, $\rho$ es la densidad del agua, $Z b$ es la cota del fondo, $\tau s$ es la fricción de la superficie 
Tabla 1. Caudales máximos mensuales desde el año de 1990 hasta el año de 2005

\begin{tabular}{lllllllllllll}
\hline & enero & febrero & marzo & abril & mayo & junio & julio & agosto & setiembre & octubre & noviembre & diciembre \\
\hline $\mathbf{1 9 9 0}$ & 2.67 & 4.059 & 5.448 & 3.165 & 2.261 & 0.439 & 0.1986 & 0.0145 & 0.2152 & 0.6903 & 0.9579 & 3.663 \\
1991 & 3.856 & 3.173 & 3.545 & 1.021 & 0.2262 & 0.05172 & 0.0286 & 0.02281 & 0.02776 & 0.2905 & 0.1599 & 0.5338 \\
1992 & 2.516 & 4.898 & 5.822 & 5.778 & 2.44 & 1.119 & 0.1182 & 0.06869 & 0.199 & 0.7036 & 1.093 & 3.168 \\
1993 & 4.688 & 7.417 & 8.782 & 7.74 & 4.371 & 1.662 & 0.5612 & 0.1314 & 0.1992 & 0.5063 & 0.3637 & 0.3224 \\
1994 & 1.207 & 1.07 & 3.13 & 2.329 & 1.368 & 0.5226 & 0.02963 & 0.05275 & 0.1039 & 0.9328 & 1.249 & 2.776 \\
1995 & 1.834 & 4.609 & 5.794 & 3.453 & 1.894 & 0.6363 & 0.05648 & 0.01503 & 0.1577 & 0.3094 & 0.5739 & 0.9918 \\
$\mathbf{1 9 9 6}$ & 1.625 & 4.177 & 2.34 & 1.819 & 0.4994 & 0.02527 & 0.00827 & 0.03749 & 0.21 & 0.4919 & 0.569 & 1.39 \\
$\mathbf{1 9 9 7}$ & 4.214 & 5.136 & 8.164 & 4.184 & 1.644 & 0.5383 & 0.04701 & 0.01921 & 0.2036 & 0.8549 & 1.272 & 1.116 \\
$\mathbf{1 9 9 8}$ & 1.94 & 8.432 & 6.228 & 5.811 & 3.057 & 1.332 & 0.268 & 0.05386 & 0.29 & 0.5421 & 1.374 & 2.268 \\
$\mathbf{1 9 9 9}$ & 5.832 & 8.364 & 7.014 & 4.431 & 2.312 & 0.8392 & 0.08835 & 0.094 & 0.2017 & 1.08 & 0.9541 & 4.794 \\
$\mathbf{2 0 0 0}$ & 5.655 & 6.876 & 8.285 & 5.031 & 2.299 & 0.7774 & 0.107 & 0.08752 & 0.3229 & 0.3521 & 1.175 & 0.8483 \\
$\mathbf{2 0 0 1}$ & 1.923 & 4.472 & 3.474 & 3.286 & 1.615 & 0.6812 & 0.06219 & 0.007904 & 0.1078 & 1.107 & 1.858 & 2.993 \\
$\mathbf{2 0 0 2}$ & 3.415 & 6.205 & 7.617 & 3.875 & 1.748 & 0.3087 & 0.0367 & 0.04028 & 0.1229 & 0.518 & 0.2919 & 1.424 \\
$\mathbf{2 0 0 3}$ & 0.6895 & 5.814 & 2.928 & 2.521 & 1.544 & 0.3701 & 0.05305 & 0.02641 & 0.242 & 0.8243 & 1.575 & 2.908 \\
$\mathbf{2 0 0 4}$ & 4.281 & 5.977 & 4.981 & 3.498 & 1.282 & 0.2651 & 0.03579 & 0.03369 & 0.1481 & 0.2804 & 0.4398 & 2.458 \\
$\mathbf{2 0 0 5}$ & 3.629 & 4.61 & 10.03 & 5.746 & 2.977 & 1.381 & 0.2383 & 0.04438 & 0.1653 & 0.28 & 1.546 & 2.024 \\
\hline
\end{tabular}

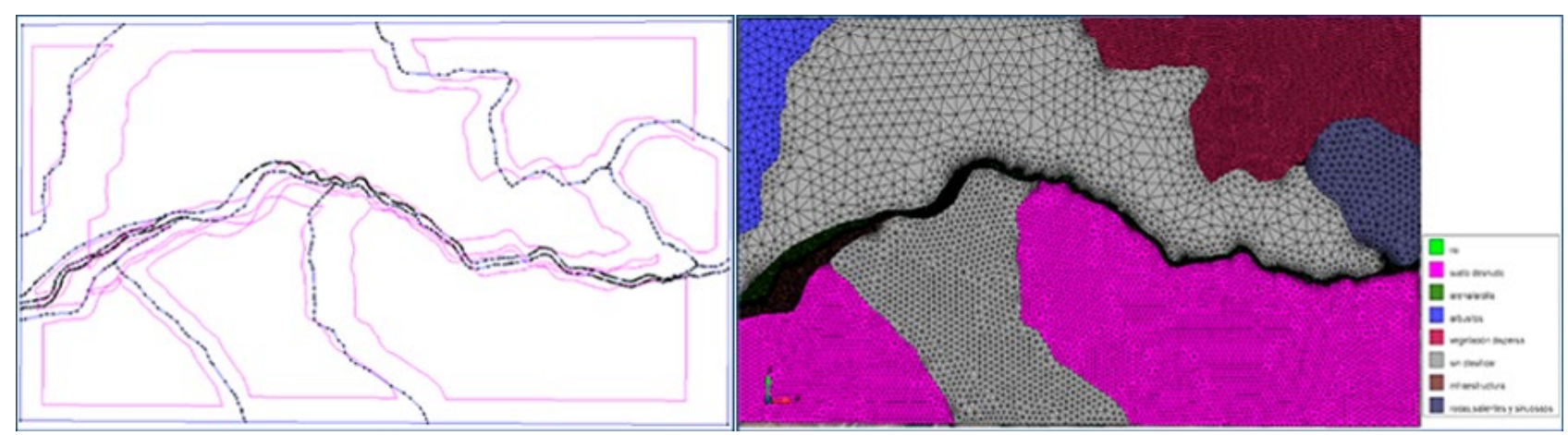

Figura 2. Digitalización importada de QGIS 3.8.2, como base para superficies NURBS, y rugosidad de los suelos.

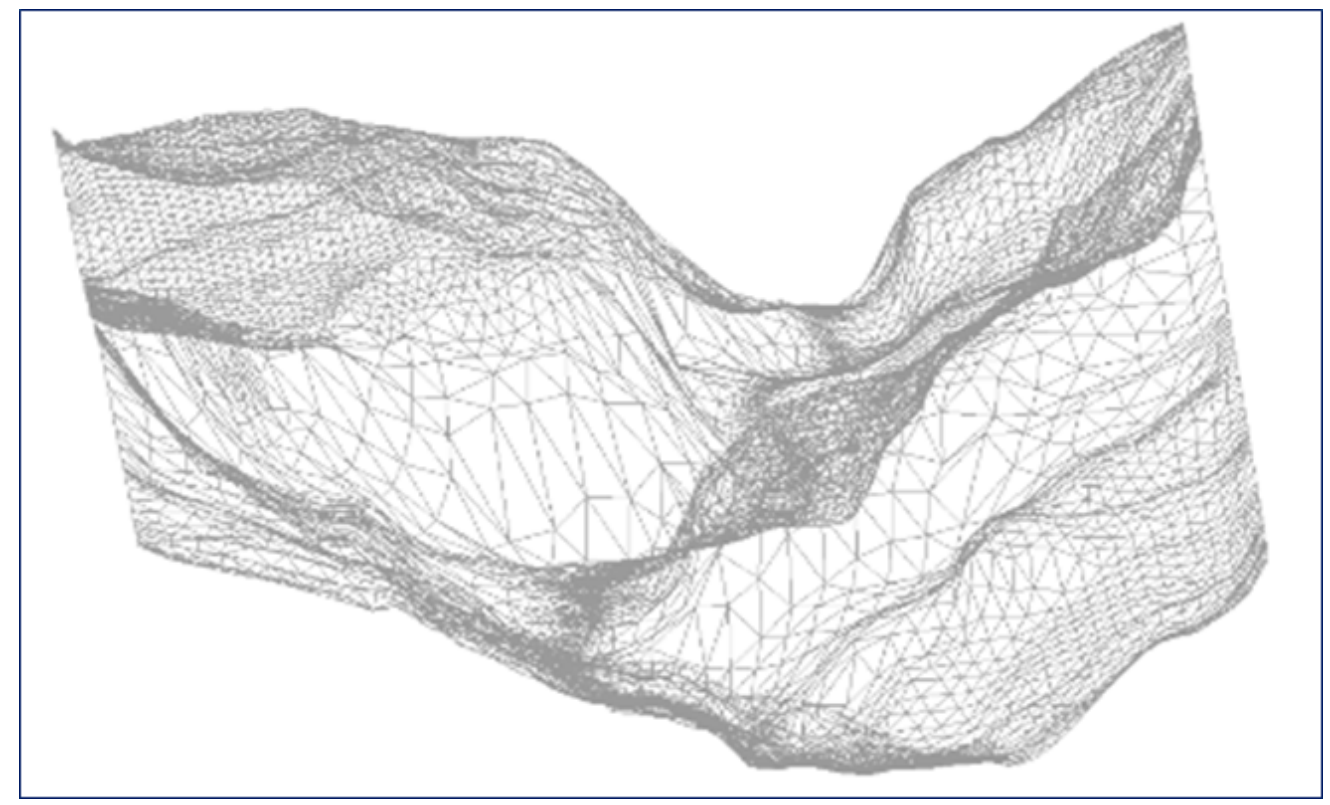

Figura 3. Modificación de la malla para estudiar el flujo del agua por secciones transversales. 
libre debida al rozamiento producido por el viento, $\tau b$ es la fricción debida al rozamiento producido del fondo y $v t$ es la viscosidad turbulenta.

$$
\begin{aligned}
& \frac{\partial h}{\partial t}+\frac{\partial h U_{x}}{\partial x}+\frac{\partial h U_{y}}{\partial y}=0 \\
& \frac{\partial}{\partial t}\left(h U_{x}\right)+\frac{\partial}{\partial x}\left(h U_{x}^{2}+g \frac{h^{2}}{2}\right)+\frac{\partial}{\partial y}\left(h U_{x} U_{y}\right) \\
& \quad=-g h \frac{\partial Z_{b}}{\partial x}+\frac{\tau_{s, x}}{\rho}-\frac{\tau_{b, x}}{\rho}+\frac{\partial}{\partial x}\left(v_{t} h \frac{\partial U_{x}}{\partial x}\right)+\frac{\partial}{\partial y}\left(v_{t} h \frac{\partial U_{x}}{\partial y}\right) \\
& \frac{\partial}{\partial t}\left(h U_{y}\right)+\frac{\partial}{\partial x}\left(h U_{x} U_{y}\right)+\frac{\partial}{\partial y}\left(h U_{y}^{2}+g \frac{h^{2}}{2}\right) \\
& \quad=-g h \frac{\partial Z_{b}}{\partial y}+\frac{\tau_{s, y}}{\rho}-\frac{\tau_{b, y}}{\rho}+\frac{\partial}{\partial x}\left(v_{t} h \frac{\partial U_{y}}{\partial x}\right)+\frac{\partial}{\partial y}\left(v_{t} h \frac{\partial U_{y}}{\partial y}\right)
\end{aligned}
$$

Figura 4. Algunas ecuaciones como base matemática del software IBER V2.5.

Fuente: Bladé et al. (2014)

\section{RESULTADOS Y DISCUSIÓN}

Como resultados, se muestra que, el caudal obtenido no posee la capacidad de producir desbordamientos en la parte alta de una cuenca (figura 5), ello es debido al encajonamiento, la geomorfología, el tipo de material geológico y amplitud del cauce del río; pero, si se observa una importante altura del nivel del agua, relacionada con la descarga rápida de flujo de agua. Sin embargo, el caudal ingresado puede afectar el cauce a una distancia de $480 \mathrm{~m}$ aproximadamente, medido desde la entrada del río, pero no se observa un desborde, lo cual está explicado por la presencia de barreras naturales geomorfológicas que dificultan el desarrollo de la inundación como lo explican Hernández-Uribe et al. (2017) (Ver figura 6).

Se analizó tres secciones transversales diferentes, para mostrar la evolución del agua en diferentes intervalos de tiempo y diferentes espacios. El tiempo tomado para su evaluación fue donde el caudal se presentó en sus picos

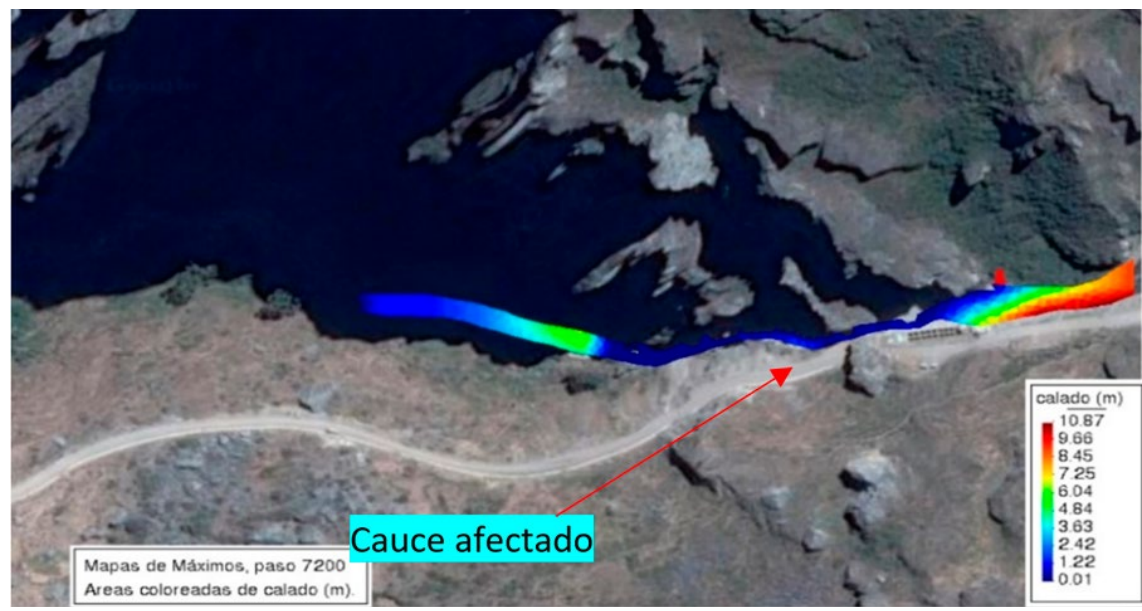

Figura 5. Primeros resultados del software IBER, no se observa desbordamientos del caudal simulado por factores físicos en la cuenca

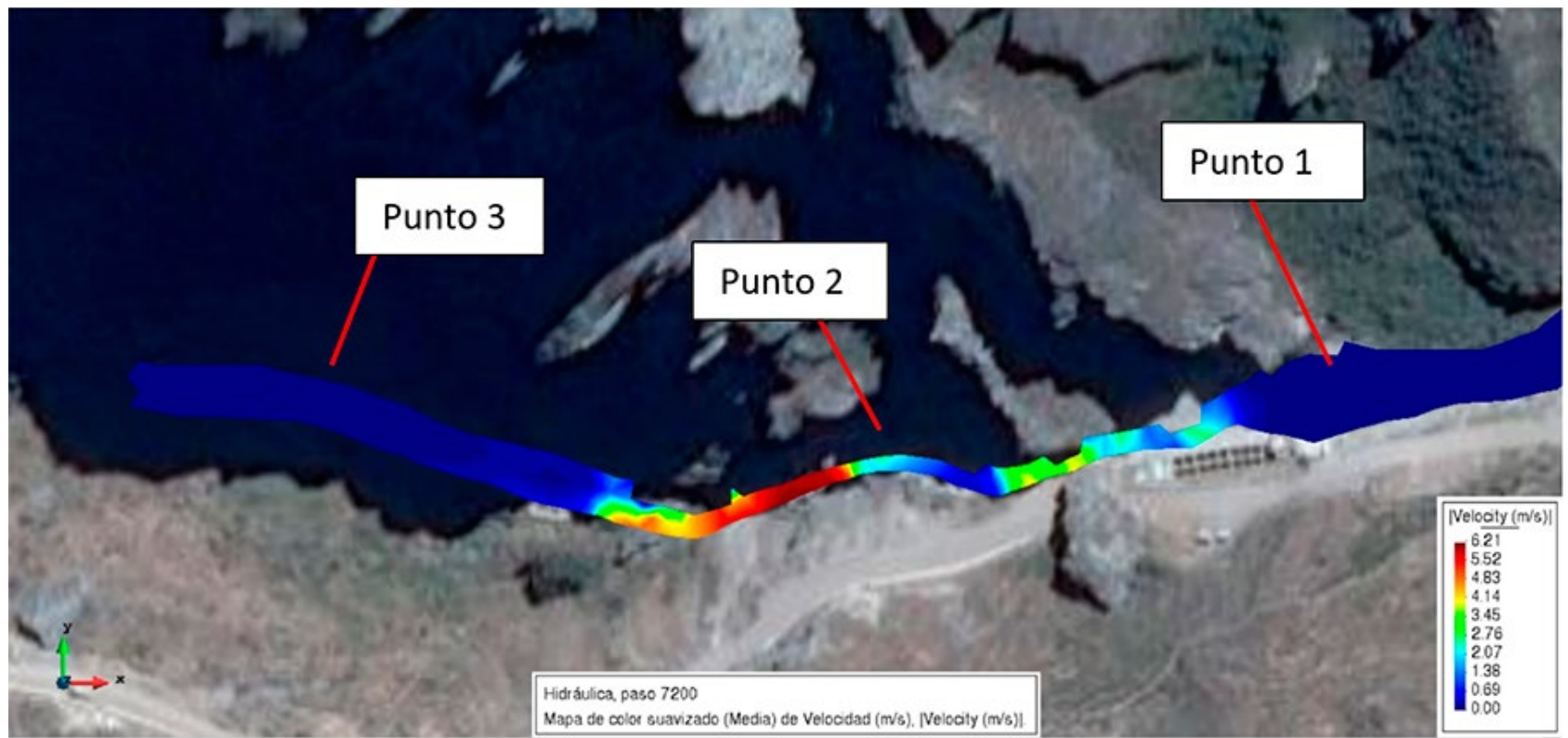

Figura 6. Puntos de análisis, visto de perfil del comportamiento de la inundación 
más altos para los tres puntos, los cuales fueron a 3780 s, 4980 s y 6420 s. Estas tres secciones transversales tienen características diferentes. En la sección transversal 01 se encuentra un pequeño reservorio a $100 \mathrm{~m}$ del inicio de la simulación, que ante un proceso de inundación alcanzaría caudal pico de $10.03 \mathrm{~m}^{3} / \mathrm{s}$; en cuanto al calado, su tirante crítico ocurre en el margen derecho con una profundidad media de $5.71 \mathrm{~m}$ alcanzando picos de 10.26 $\mathrm{m}$ de profundidad, debido a la acumulación del agua en dicho reservorio, que impide el normal paso del agua, a través de su curso; por lo que el curso de la inundación y el efecto del mismo, está regulado por la geomorfología característica de las zonas de alta montaña o cabeceras de cuenca (Alcántara Boza, 2019) (Ver figura 7).

\subsection{Segundo punto de evaluación}

Este punto se evaluó a una distancia de $230 \mathrm{~m}$, desde el punto de entrada de agua en el área estudiada y a una distancia de $130 \mathrm{~m}$ desde el primer punto evaluado. Su sección trasversal presenta una topografía de pendiente abrupta en el cauce del río. En la simulación se observar que el flujo presenta una velocidad rápida en periodos cortos. El río tiene un ancho promedio de $1.5 \mathrm{~m}$, además se puede observar que la zona no es inundable; el intervalo de tiempo evaluado fue de $4980 \mathrm{~s}$, tiempo donde alcanza su máximo pico. Es importante considerar el amortiguamiento que recibe el flujo de agua por el tipo de espacio, ayudado por las características geomorfológicas, los tipos de suelo y la vegetación, que convergen en la retención del flujo de agua lo cual va a contribuir favorablemente para el siguiente tramo.
El calado en este segundo punto alcanza una altura de tirante de $1.18 \mathrm{~m}$ de profundidad del flujo, por tanto, no ocurre un tirante crítico (figura 8), esta evaluación se hizo para un intervalo de tiempo de $3810 \mathrm{~s}$. Su velocidad máxima alcanza $4.21 \mathrm{~m} / \mathrm{s}$ el cual se debe principalmente a la pendiente alta, la geomorfología y el material rocoso en ambos lados del cauce.

En cuanto al froude, alcanza un coeficiente de 4.06 en el intervalo de tiempo de $4980 \mathrm{~s}$, el cual refiere a un flujo crítico-muy crítico, lo cual obedece a un cauce en forma de $\mathrm{V}$ que geomorfológicamente se explica como un tránsito muy rápido de un valle en forma de $\mathrm{U}$ a un valle en forma de V, por la erosión, (figura 8).

\subsection{Evaluación del tercer punto}

El tercer punto se encuentra aproximadamente a $390 \mathrm{~m}$ desde la entrada del flujo al área evaluado y a una distancia de $160 \mathrm{~m}$ del segundo punto. Este punto presenta una topografía suave en ambos lados del cauce, (figura 9), con un ancho medio de $3 \mathrm{~m}$, por lo que no presenta una inundación importante; empero el punto evaluado tiene un alto riesgo a inundación si se presentasen precipitaciones pino y de larga duración.

El calado, en este punto, alcanza $1.31 \mathrm{~m}$ de profundidad, por tanto, presenta un flujo supercrítico o rápido, lo cual se explica por el tipo de topografía cambiante, si bien no es abrupto, pero presenta ondulaciones. Sin embargo, la velocidad alcanza $3.5 \mathrm{~m} / \mathrm{s}$ para un intervalo de tiempo de 6
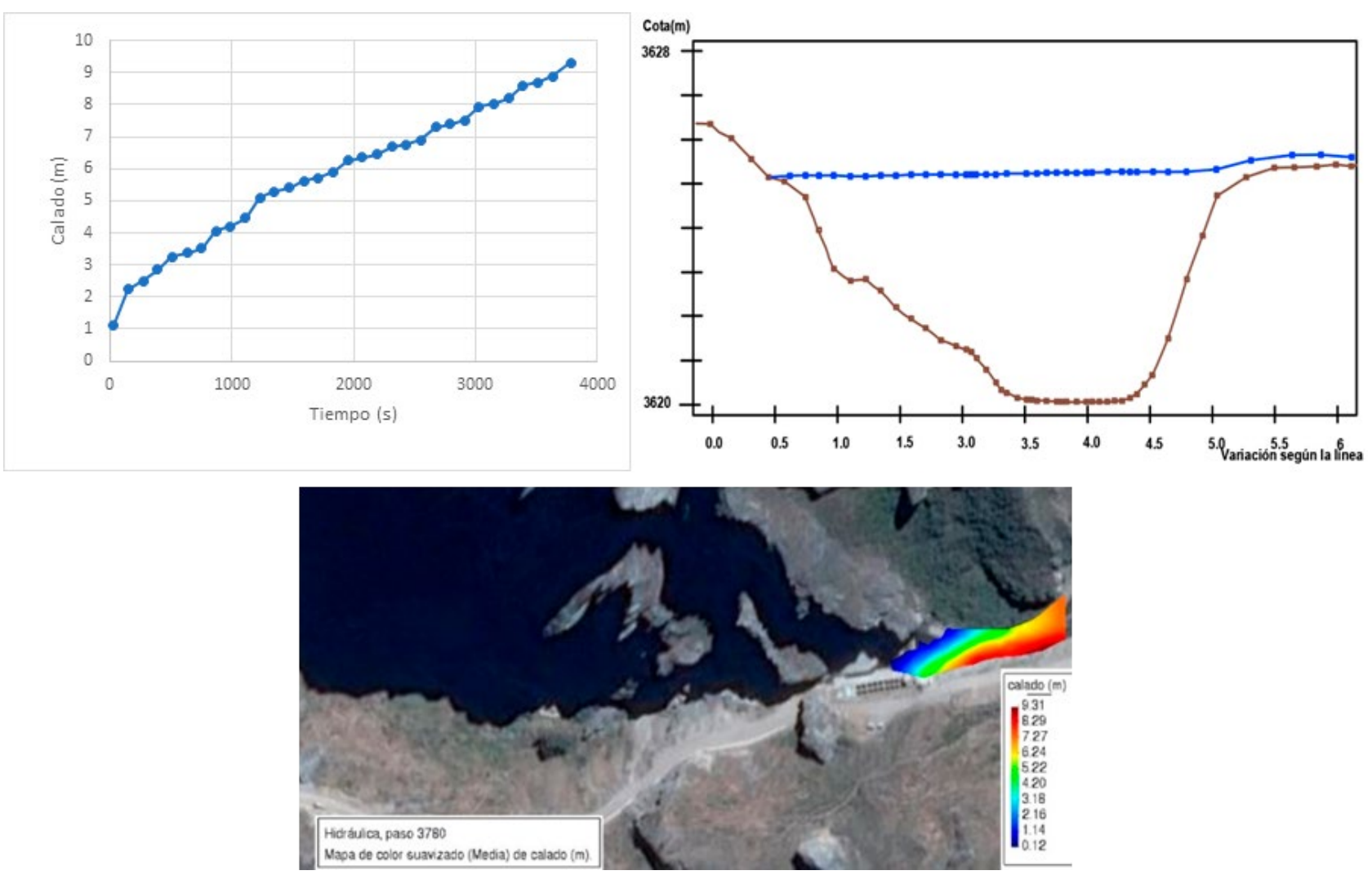

Figura 7. Comportamiento de la inundación en el punto 1 de la figura 6 

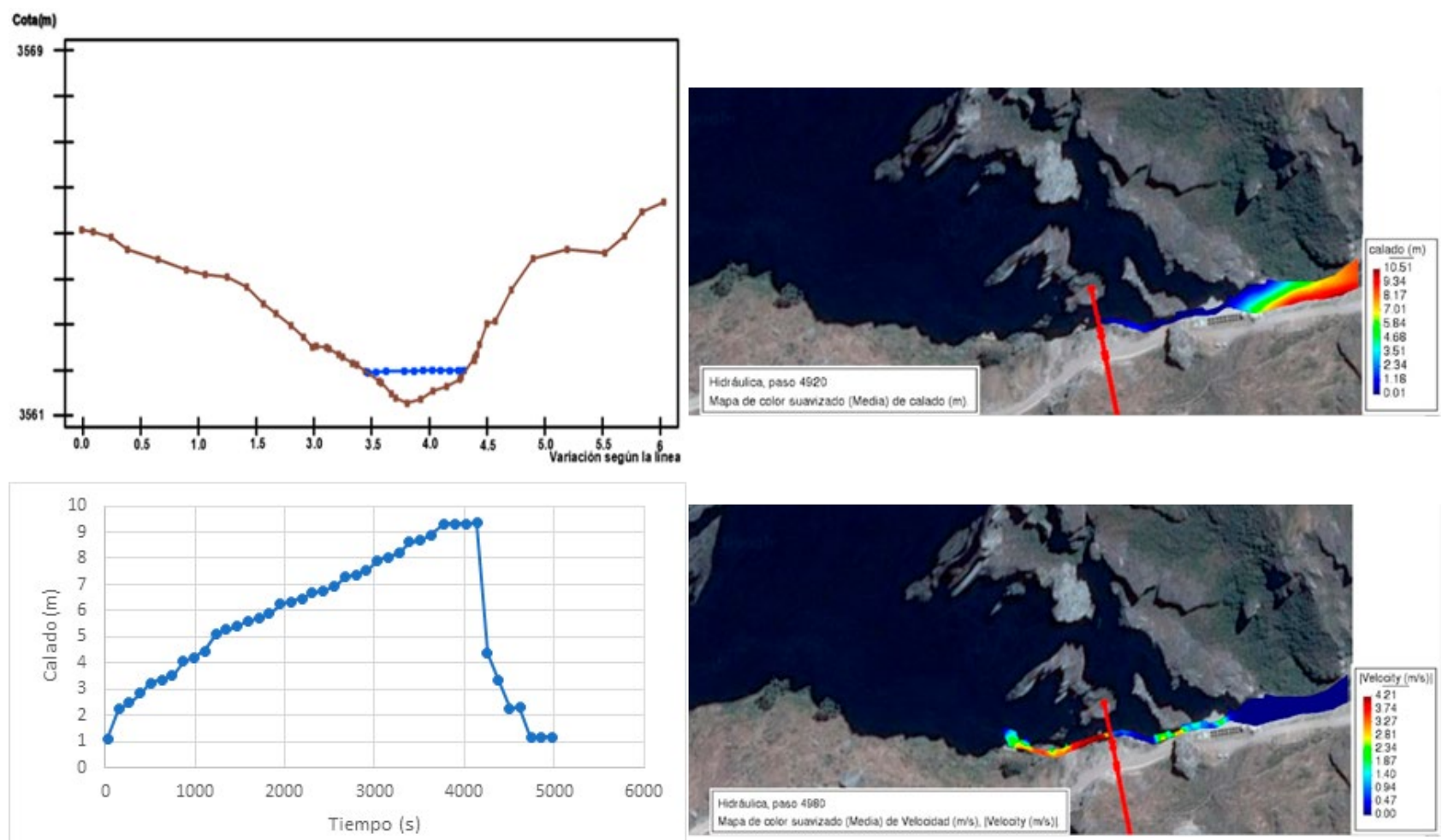

Figura 8. Geomorfología del terreno, observación del calado y dimensiones del río.
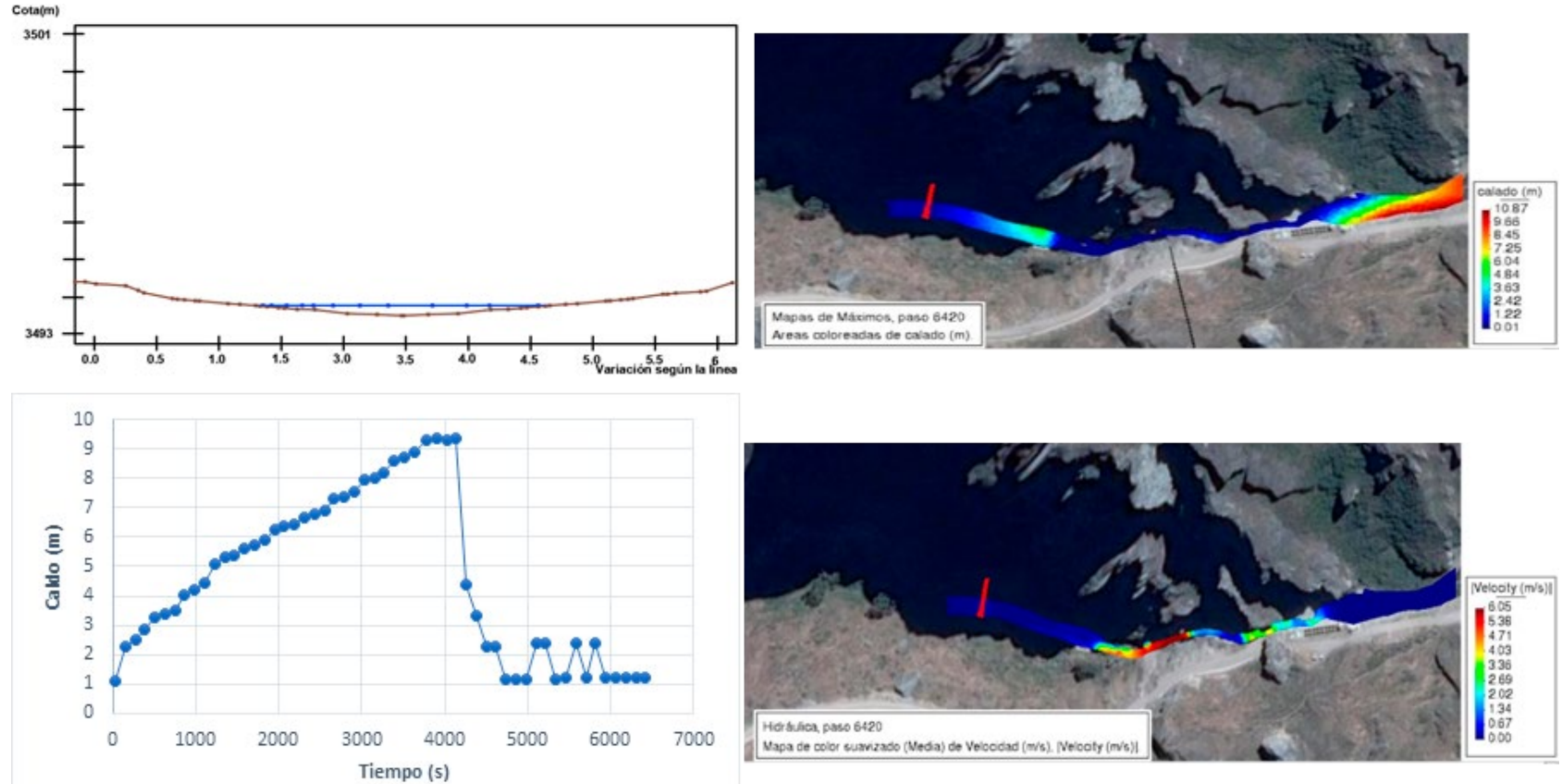

Figura 9. Evaluación de la inundación en el tercer punto

$420 \mathrm{~s}$ al igual que el calado, la velocidad también depende de la topografía y pendiente de la zona de estudio.

\subsection{Dirección de velocidades a lo largo del cauce del río simulado}

Para el periodo de evaluación de 15 años, $(1990$ - 2005, periodo de data), durante los primeros 100 metros no ocurre un fuerte movimiento de agua (figura 10-a), esto debido a las características topográficas moderadas y suave, salvo en la salida y en la entrada del reservorio, las velocidades que afectan las diferentes partes del reservorio son mínimos, alcanzando $0.07 \mathrm{~m} / \mathrm{s}$, el cual se debe a que el agua acumulada, genera un amortiguamiento. En los siguientes $130 \mathrm{~m}$, (figura 10-b), encontramos tramos donde el flujo es afectado 


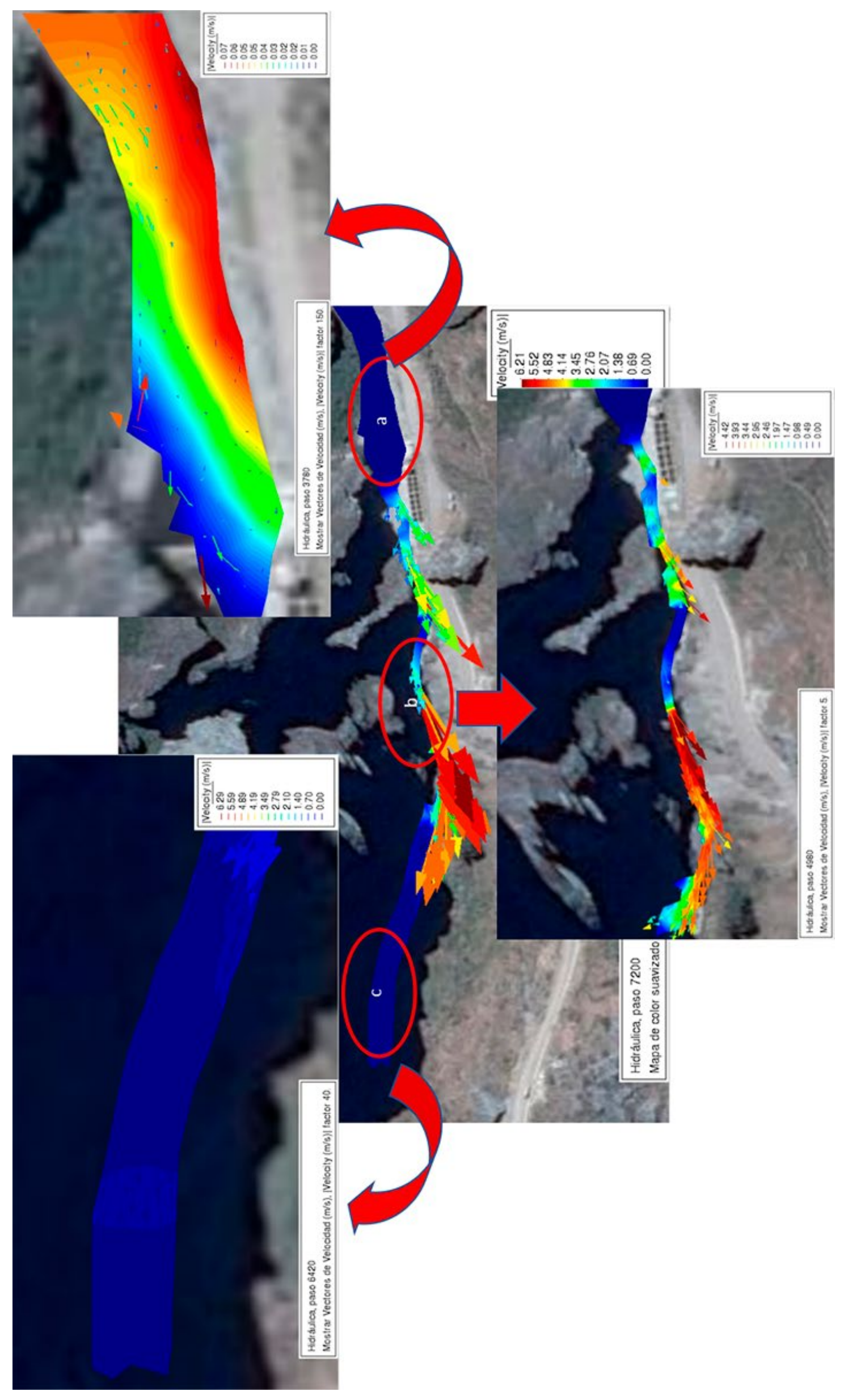

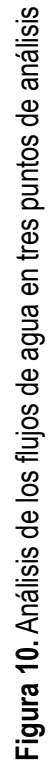


por el cauce del río; el principal factor es la pendiente, fuertemente inclinada, lo cual difiere de los procesos de inundación en partes planas, como lo muestran Martínez et al. (2017) y Raquel Martínez-Cantó \& Arturo Hidalgo (2019). La dirección más afectada es el margen izquierdo, el cual puede generar una fuerte erosión y transporte de materiales, alcanzando una velocidad de $4.21 \mathrm{~m} / \mathrm{s}$, sin duda este punto sería el más afectado. En cuanto al último tramo, su velocidad máxima alcanza $3.5 \mathrm{~m} / \mathrm{s}$ y en algunos casos disminuye hasta $1.40 \mathrm{~m} / \mathrm{s}$ es decir, este último tramo si bien sus velocidades son medianamente altas, no presenta una erosión, lo cual estaría explicado por el amortiguamiento de la misma superficie y vegetación herbáceas que retienen el flujo de agua en el tiempo de $7200 \mathrm{~s}$.

Se discute, también, que el flujo de agua no llega a salir del área simulada, por la forma de la microcuenca, topografía cerrada, pendientes muy inclinadas, plantas herbáceas y arbustivas que retienen el agua, el desarrollo evolutivo del suelo de morrénico a leptosoles, entre otros. Al momento de evaluar, una de las variables más influyentes es la gran pérdida del flujo debido a la infiltración, puesto que el área avaluado presenta un material arenoso asociado a vegetación y la capacidad de retención de agua, característico de las partes más altas de las cuencas; un factor importante también son las características de materiales genéticos morrénicos, puesto que el promedio de la infiltración de las microcuencas baños, minascocha y Quilla presenta una tasa de infiltración de $10 \mathrm{~cm} / \mathrm{h}$.

\section{CONCLUSIONES}

En cabeceras de cuenca, los flujos de agua no llegan a salir de su cauce, lo cual se debe básicamente a las características geomorfológicas en forma de U e inicios de V; sin embargo, en el área de estudio, el caudal máximo que ingresa es suficiente para poder cubrir superficies medianamente onduladas, como el caso del reservorio que se encuentra a $100 \mathrm{~m}$ de distancia desde la entrada del flujo, inundándolo, sin generar mayores desastres, donde la velocidad del flujo se reduce a $0.07 \mathrm{~m} / \mathrm{s}$.

La velocidad es directamente proporcional a la pendiente $\mathrm{y}$ es inversamente proporcional al tirante (calado). La dirección de velocidad a la salida de cabeceras de cuencas tiende a ser lineal, alcanzando velocidades de $4.21 \mathrm{~m} / \mathrm{s}$ en pendientes relativamente abruptas; y en pendientes suaves, el flujo del agua alcanza velocidades de hasta $1.40 \mathrm{~m} / \mathrm{s}$.

En cuanto a la etapa de pre-proceso del software IBER y en cabeceras de cuenca, se debe puntualizar la sectorización y asignación adecuada del coeficiente de Manning, así como la generación y modificación de la malla, ello, por las características geomorfológicas encajonadas, el tipo de macizos rocosos y acumulado sedimentario morrénico a leptosoles.

\section{REFERENCIAS}

Alcántara Boza, A. (2019). Influencia de la Geomorfología y la Meteorización en la Humedad del suelo y recargas de agua en la cabecera de cuenca del río Jequetepeque. In Revista del Instituto de Investigación de la Facultad de Ingeniería Geológica, Minera, Metalúrgica y Geográfica (Vol. 22, Issue 43). https://doi.org/10.15381/iigeo.v22i43.16694

Areu-Rangel, O. S., González-Cao, J., Crespo, A. J. C., \& Bonasia, R. (2019). Numerical modelling of hydrological safety assignment in dams with IBER. Sustainable Water Resources Management, 5(1), 347-358. https://doi. org/10.1007/s40899-017-0135-2

Bladé, E., Cea, L., Corestein, G., Escolano, E., Puertas, J., Vázquez-Cendón, E., Dolz, J., \& Coll, A. (2014). Iber: herramienta de simulación numérica del flujo en ríos. Revista Internacional de Metodos Numericos Para Calculo y Diseno En Ingenieria, 30(1), 1-10. https://doi. org/10.1016/j.rimni.2012.07.004

Calzolari, C., Ungaro, F., \& Vacca, A. (2021). Effectiveness of a soil mapping geomatic approach to predict the spatial distribution of soil types and their properties. Catena, 196. https://doi.org/10.1016/j.catena.2020.104818

Gil, C., \& Villanueva, I. (2010). Efectos de la cartografía sobre la modelización hidráulica bidimensional de crecidas. Conference: II Jornadas Ingenieria AguaAt: Barcelona, 2006. https://www.researchgate.net/ publication/323991827_Efectos_de_la_cartografia sobre_la_modelizacion_hidraulica_bidimensional_de crecidas

Hernández-Uribe, R. E., Barrios-Piña, H., \& Ramírez, A. I. (2017). Análisis de riesgo por inundación: Metodología y aplicación a la cuenca Atemajac. Tecnologia y Ciencias Del Agua, 8(3), 5-25. https://doi.org/10.24850/j-tyca-2017-03-01

INGEMMET. (2020). Carta Geológica Nacional. Escala 1/100 $000 . \quad \mathrm{https}: / / \mathrm{www}$.ingemmet.gob.pe/carta-geologicanacional-escala-1-100-000

Martínez-Cantó Raquel, \& Hidalgo Arturo. (2019). A Methodology Based on Numerical Simulation to Study River Floods. Application to Lower River Omaña Basin. Water Resources, 46(6), 844-852. https://doi.org/10.1134/ S0097807819060150

Martínez, R., Martínez, A., \& Hidalgo, A. (2017). Modelo bidimesional para flujo en aguas poco profundas: tratamiento con iber. July. https://www.researchgate.net/ publication/318317258_Modelo_bidimesional_para_flujo_ en_aguas_poco_profundas_tratamiento_con_iber

Martins, M. M. C., Gomes, A. A. T., \& Santos, P. P. (2019). Delimitation of flood-prone areas in the urban center of amarante, north of Portugal, using the Iber software. Revista Brasileira de Geomorfologia, 20(1), 185-200. https://doi. org/10.20502/rbg.v20i1.1407

O'Connor, J. E., Baker, V. R., Waitt, R. B., Smith, L. N., Cannon, C. M., George, D. L., \& Denlinger, R. P. (2020). The Missoula and Bonneville floods-A review of ice-age megafloods in the Columbia River basin. Earth-Science Reviews, 103181. https://doi.org/10.1016/j.earscirev.2020.103181

Ochoa, N., Gomez, O., Sanchez, S., \& Delgado, R. (1999). Diversidad de diatomeas y dinoflagelados marinos del Perú. Boletín Del Instituto Del Mar Del Perú, 18, 1-14. http:// biblioimarpe.imarpe.gob.pe/handle/123456789/975

Poma, W., \& Alcántara, G. H. (2011). Estudio de suelos y capacidad de uso mayor del departamento de cajamarca (p. 81). 
Gobierno Regional de Cajamarca. http://geoservidorperu. minam.gob.pe/geoservidor/Archivos/Mapa/Cajamarca/ Memoria_Descriptiva_Suelos_CUM.pdf

Rivera-Trejo, F., Uh-Us, F., Soto-Cortés, G., \& Díaz-Flores, L. L. (2013). Inspección de cauces: Guía de reconocimiento en campo. Tecnologia y Ciencias Del Agua, 4(2), 149-161. http://www.redalyc.org/articulo.oa?id=353531982010

Rocha, A. (2013). Introducción a la Hidráulica de las Obras Viales Arturo Rocha Felices. In Instituto de la Construccion y Gerencia: Vol. Tercera Ed (Issue Fondo Editorial ICG). www.construccion.org
Sanz-Ramos, M., Olivares, G., \& Bladé, E. (2017). Simulación de rotura de presas aterradas con fondo móvil. Caso de estudio. V Jornadas de Ingeniería Del Agua. http://www.iberaula.es/ Temas/DisplayTema?id_tema $=707$

Sarango, D., Velásquez, T., Rozas, G., \& Gástelo, J. (2018). Estudio de máximas avenidas para la protección de zonas de posible inundación ubicada en el tramo La Oroya- $40 \mathrm{~km}$ aguas abajo Cuenca del Río Mantaro. Revista Del Instituto de Investigación de La Facultad de Ingeniería Geológica, Minera, Metalurgica y Geográfica, 20(39), 27-38. https:// revistasinvestigacion.unmsm.edu.pe/index.php/iigeo/ article/view/14163 